\title{
Erratum: the energy of the analytic lump solution in SFT
}

\author{
L. Bonora, ${ }^{a, b, c}$ S. Giaccari ${ }^{a, b}$ and D.D. Tolla ${ }^{d}$ \\ ${ }^{a}$ International School for Advanced Studies (SISSA), \\ Via Bonomea 265, 34136 Trieste, Italy \\ ${ }^{b}$ INFN, Sezione di Trieste, \\ Trieste, Italy \\ ${ }^{c}$ Yukawa Institute for Theoretical Physics, Kyoto University, \\ Kyoto 606-8502, Japan \\ ${ }^{d}$ Department of Physics and University College, Sungkyunkwan University, \\ Suwon 440-746, South Korea \\ E-mail: bonora@sissa.it, giaccari@sissa.it, ddtolla@skku.edu
}

ERRATUM TO: JHEP08(2011)158

After this paper was published in JHEP, a reconsideration of all the problems tackled in it led us to [2]. In the latter we confirm all the results of this paper as well as of [1], but we correct the interpretation of $\epsilon$ as a gauge parameter contained in section 7.2 and in the last paragraph of 9.2. In [2] we provide evidence that $\left\langle\psi_{\epsilon}, \psi_{\epsilon} \psi_{\epsilon}\right\rangle^{(s)},\left\langle\psi_{u}, \psi_{\epsilon} \psi_{\epsilon}\right\rangle^{(s)}$ and $\left\langle\psi_{\epsilon}, \psi_{u} \psi_{u}\right\rangle^{(s)}$ all depend on $\epsilon$. Consequently $\epsilon$ is simply a regulator and cannot be interpreted as a gauge parameter. The only meaningful results are obtained in the limit $\epsilon \rightarrow 0$. Concerning the claim that "The obstruction to integrating by part is the UV divergence or the corresponding subtraction, which ... are $\epsilon$-independent." in the last paragraph of section 9.2, it is true, but this does not lead by itself to the implicit conclusion that one can integrate by part the expression $\left\langle\psi_{\epsilon}, Q \psi_{u}\right\rangle-\left\langle\psi_{u}, Q \psi_{\epsilon}\right\rangle$ and get 0 , because the UV subtraction is applied to the three-points correlators, not to the string field $\psi, \psi_{\epsilon}$, to which $Q$ applies.

\section{References}

[1] L. Bonora, S. Giaccari and D.D. Tolla, Analytic solutions for Dp branes in SFT, JHEP 12 (2011) 033 [arXiv:1106.3914] [INSPIRE].

[2] L. Bonora, S. Giaccari and D.D. Tolla, Lump solutions in SFT. Complements, expanded version, arXiv:1109.4336 [inSPIRE]. 